\title{
Non-invasive monitoring of cisplatin and erlotinib efficacy against lung cancer in orthotopic SCID mouse models by small animal FDG-PET/CT and CT
}

\author{
TAMAKI OTANI ${ }^{1}$, KAZUYA KONDO ${ }^{2}$, HIROMITSU TAKIZAWA ${ }^{3}$, KOICHIRO KAJIURA ${ }^{3}$, \\ HARUHIKO FUJINO $^{3}$, HIDEKI OTSUKA ${ }^{4}$ and HIROKAZU MIYOSHI ${ }^{1}$
}

\author{
${ }^{1}$ Radioisotope Research Center, Tokushima University Graduate School; ${ }^{2}$ Department of Oncological Medical Services, \\ Institute of Biomedical Sciences, Tokushima University Graduate School; ${ }^{3}$ Department of Thoracic, \\ Endocrine Surgery and Oncology, Institute of Biomedical Sciences; \\ ${ }^{4}$ Department of Medical Imaging/Nuclear Medicine, Institute of Biomedical Sciences, \\ Tokushima University Graduate School, Tokushima City, Tokushima 770-8503, Japan
}

Received April 5, 2018; Accepted October 12, 2018

DOI: $10.3892 /$ or.2018.6818

\begin{abstract}
We established patient-like models of lung cancer metastasis by orthotopically implanting human non-small cell lung cancer cell lines into SCID mice. We evaluated the utilities of small-animal computed tomography (CT) and positron-emission tomography-computed tomography (PET/CT) in these models to non-invasively and repeatedly monitor the anticancer effects of cisplatin and erlotinib. We orthotopically implanted three non-small cell lung cancer cell lines, A549, FT821 and PC-9, into SCID mice. These mice were then divided into three groups: Control, cis-diamminedichloroplatinum (II) (CDDP) (7-mg/kg CDDP, single administration intraperitoneally), and erlotinib (25 $\mathrm{mg} / \mathrm{kg}$ erlotinib/day, oral administration 5 days/week). After treatment initiation, we repeatedly performed $\mathrm{PET} / \mathrm{CT}$ and $\mathrm{CT}$ measurements and assessed anticancer effects based on tumor volumes and FDG uptake. A549 tumors were not affected by CDDP or erlotinib. FT821 tumors were highly responsive to CDDP. PC-9 tumors, which have an epidermal growth factor receptor mutation, were highly responsive to erlotinib. Histological results and metastatic rates correlated with the anticancer effects shown by CT. In our orthotopic SCID mouse lung cancer models, ${ }^{18}$ FDG-PET/CT and CT imaging non-invasively and repeatedly monitored the efficacies of cisplatin and erlotinib against
\end{abstract}

Correspondence to: Professor Kazuya Kondo, Department of Oncological Medical Services, Institute of Biomedical Sciences, Tokushima University Graduate School, 3-18-15 Kuramotocho, Tokushima City, Tokushima 770-8503, Japan

E-mail: kzykondo@tokushima-u.ac.jp

Key words: lung cancer, orthotopic model, positron emission tomography/computed tomography (PET/CT), $\quad\left[{ }^{18} \mathrm{~F}\right]$ fluorodeoxyglucose not only implanted tumors, but also mediastinal lymph node metastases.

\section{Introduction}

Lung cancer is the most common cause of cancer death in Japan, North America and Europe. Approximately $85 \%$ of these cases are non-small cell lung cancer (NSCLC) (1). More than $50 \%$ of patients with NSCLC have lymphadenopathy and/or distant metastasis and, thus, a poor prognosis. The 5-year relative survival rate of patients with distant metastases is less than $4 \%$ (2). The primary reason for the difficulties associated with treating NSCLC is that patients are identified at a very late stage and effective treatments available for advanced NSCLC are limited. However, advances have recently been achieved in elucidating the molecular origins of NSCLC, with the better classification of histological subtypes and development of targeted therapies. A molecular analysis of NSCLC has suggested many new molecularly targeted drugs for its treatment $(3,4)$. Thus, suitable preclinical tumor models are required to assess whether new anticancer drugs will be effective for NSCLC.

Preclinical tumor models are a fundamental component for the study and design of new treatment regimens for cancer (5). These models are either ectopic (tumors in an abnormal site, such as lung cancer grown subcutaneously) or orthotopic (tumors in their organ or tissues of origin, such as lung cancer in the lung). In 1889, Paget proposed the 'seed and soil' theory in which an organ-specific site provides tumor cells with the most appropriate environment for local growth and metastasis (6). Subcutaneously implanted tumors in mice generally grow rapidly, which does not reflect the slower doubling times of most human cancers; therefore, they may be more sensitive to chemotherapy drugs than orthotopic tumors (7). Models with subcutaneously implanted tumors generally show a low incidence of distant metastatic disease. The orthotopic transplantation of tumors results in a higher incidence of distant metastases (8), and these models show spontaneous metastasis. 
Orthotopic models may provide more relevant pharmacokinetic and pharmacodynamic information than subcutaneous ectopic models (9). The advantage of orthotopic models is their similar characteristics to those of tumors in clinical settings (5).

Considerable efforts have focused on developing clinically relevant models using orthotopic tumor implantation. We established an orthotopically implanted lung cancer model in SCID mice without thoracotomy (10-15). We previously reported an animal model that consisted of the orthotopic implantation of lung cancer cell lines and showed a high frequency of lymphatic metastasis. This model was simple and reproducible, enabling transplantation in many mice at once, and metastatic patterns, exhibiting lymphatic metastases and/or distant metastases at high frequencies, were similar to those observed clinically in human lung cancer. However, the primary disadvantage of orthotopic models is that changes in tumor sizes are difficult to monitor continuously and reproducibly, and may only be assessed at necropsy.

To conduct continuous monitoring, we employed in vivo imaging techniques, such as computed tomography (CT) and positron-emission tomography-computed tomography (PET/CT), for small animals. CT and ${ }^{18} \mathrm{~F}$-fluorodeoxyglucose $\left({ }^{18} \mathrm{~F}-\mathrm{FDG}\right) \mathrm{PET} / \mathrm{CT}$ are excellent methods for detecting primary lesions or lymph node metastases in patients with NSCLC. CT provides high anatomical resolution criteria for the detection of abnormal lymph nodes, with an axial short-axis diameter of 1-cm sensitivity (51-64\%) and specificity (74-86\%). PET using ${ }^{18} \mathrm{~F}$-FDG is superior for detecting lymph node metastasis with a sensitivity of $74 \%$ and specificity of $85 \%$ (16-18). We previously reported the non-invasive monitoring of the anticancer effects of cisplatin (CDDP) on lung cancer in an orthotopic SCID mouse model using ${ }^{18} \mathrm{~F}-\mathrm{FDG}$ PET/CT (19). The findings obtained supported the use of ${ }^{18} \mathrm{~F}-\mathrm{FDG}-\mathrm{PET} / \mathrm{CT}$ to detect tumor progression and the therapeutic responses of lung cancer in an orthotopic model through non-invasive and repeated monitoring. We consider our orthotopic models to provide suitable systems for the preclinical evaluation of the antitumor efficacies of potential new NSCLC therapies.

In the present study, we evaluated the usefulness of small-animal PET/CT and CT to non-invasively and repeatedly monitor the inhibitory effects of the conventional anticancer agents, CDDP and erlotinib, on orthotopically implanted lung cancer in SCID mice. The aim of the present study was to establish a standard model to evaluate the efficacies of novel treatment regimens in lung cancer.

\section{Materials and methods}

Animals. Eighty male SCID mice (CB-17/Icr-scidJc1; CLEA Japan, Inc., Tokyo, Japan) at 6-8 weeks of age were used in the present study and maintained at the Laboratory for Animal Experiments of our institution. The animals were housed in microisolator cages on a layer of wood shavings at a temperature of $22 \pm 2^{\circ} \mathrm{C}$ under a fixed $12 \mathrm{~h}$ light/dark regime. The basic diet (MF; Oriental Yeast Co., Ltd., Tokyo, Japan) and water were available ad libitum. All experiments were performed in accordance with the guidelines established by the Tokushima University Committee on Animal Care and Use. At the end of each in vivo experiment, mice were anesthetized with isoflurane and euthanized humanely by dislocating the vertebrae.
All experimental protocols were reviewed and approved by the Animal Research Committee of The University of Tokushima, Japan.

Cell lines. We used three types of NSCLC cell lines in the present study: A549 (human adenocarcinoma lung cancer cells), FT821 (human large cell lung cancer cells) and PC-9 (human adenocarcinoma cells). We established the FT821 cell line using a primary culture of surgically resected tissue provided by Dr Haruhiko Fujino (Tokushima University, Tokushima, Japan) (13). A549 (JCRB0076) cells were purchased from the Health Science Research Resources Bank (Osaka, Japan). PC-9 (CVCL_B260) cells were kindly provided by Professor Seiji Yano (Division of Medical Oncology, Cancer Research Institute, Kanazawa University, Kanazawa, Japan) (20). PC-9 cells have an epidermal growth factor receptor (EGFR) mutation (deletion in exon 9 of the EGFR gene, del E746_A750), whereas the other two cell lines do not. These cell lines were cultured in RPMI-1640 medium (Sigma-Aldrich; Merck KGaA, Darmstadt, Germany) supplemented with $10 \%$ heat-inactivated fetal bovine serum (FBS; BioWhittaker; Lonza, Walkersville, $\mathrm{MD}, \mathrm{USA}$ ) and were maintained at $37^{\circ} \mathrm{C}$ in a humidified incubator equilibrated with $5 \% \mathrm{CO}_{2}$ and $95 \%$ air.

Orthotopic intrapulmonary implantation. As described in our previous studies (10-15), mice were fully anesthetized with $1.5 \%$ isoflurane inhalation and placed in the right lateral decubitus position with all four limbs restrained. A 1-cm transverse incision was made in the left lateral skin just below the inferior border of the scapula in each mouse. The muscles were separated from the ribs by sharp dissection and the intercostal muscles exposed. The left lung was then visible through the intercostal muscles. A 30-gauge needle was inserted $\sim 5 \mathrm{~mm}$ into the lungs through the intercostal muscle and an inoculum of $2 \times 10^{6}$ tumor cells $/ \mathrm{ml}$ with $400 \mathrm{mg} / \mathrm{ml}$ Matrigel (Collaborative Biomedical Products, Bedford, MA, USA) was then dispersed into the left lung in a final volume of $10 \mu \mathrm{l}$ medium $\left(2 \times 10^{4}\right.$ cells). The procedure required $\sim 1 \mathrm{~min}$ for completion and was easily performed. The skin incision was closed with 3-0 silk.

${ }^{18}$ F-FDG PET/CT measurements. On day 20 after the implantation of A549 cells, we performed a PET/CT measurement on each mouse. Mice were then divided into two groups: A control group [ $\mathrm{n}=8$, saline solution $(0.7 \mathrm{ml})$, single administration intraperitoneally] and cis-diamminedichloroplatinum (II) (CDDP) (Pfizer Japan Inc., Tokyo, Japan) group [n=8, $7 \mathrm{mg} / \mathrm{kg}$ CDDP $(0.7 \mathrm{ml})$, single administration intraperitoneally]. After treatments, PET/CT measurements were evaluated every 10 days until day 50 after implantation.

All scans were performed with a Siemens Inveon small-animal PET scanner (Siemens Healthcare, Knoxville, TN, USA). Mice with the orthotopic implantation of A549 cells to be monitored by ${ }^{18} \mathrm{~F}$-FDG PET/CT were fasted for $18-24 \mathrm{~h}$, with access to water only. Body weights were measured and mice were anesthetized by $1.5-2.0 \%$ isoflurane inhalation and injected via a tail-vein catheter with $10 \mathrm{MBq} / 0.1-0.2 \mathrm{ml}$ ${ }^{18} \mathrm{~F}-\mathrm{FDG}$. The lung field was scanned by $\mathrm{CT}$ [field of view (FOV): $32.0 \times 32.0 \times 48.1 \mathrm{~mm}^{3}$ ]. PET data were acquired for 20 min following a delay of 40 min to allow for FDG uptake. 
A
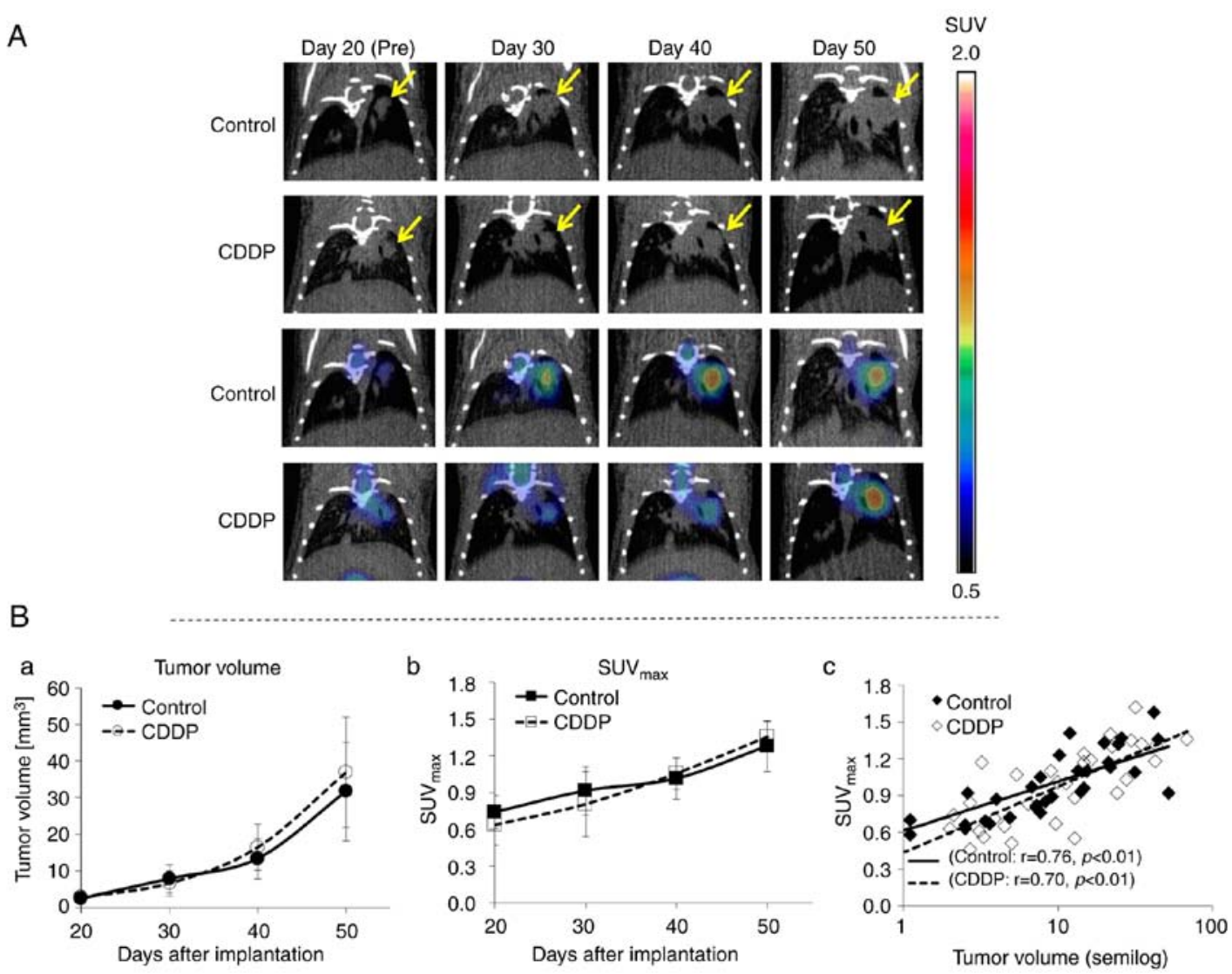

Figure 1. (A) Coronal view of CT and PET/CT images revealed an A549 tumor in the left lungs of control and CDDP mice. Yellow arrows indicate lung tumors. (B) PET/CT imaging quantitative data in mice implanted with A549 cells. a, Tumor volume; $\mathrm{b}, \mathrm{SUV}_{\text {max }}$; and c, the correlation between tumor volumes and $\mathrm{SUV}$ max

Measurement of tumor volumes by small animal CT. All scans were performed with a Siemens Inveon small-animal CT scanner (Siemens Healthcare). Mice with the orthotopic implantation of A549, FT821, or PC-9 cells were weighed and then anesthetized by $1.5-2.0 \%$ isoflurane inhalation. CT acquisition was performed as described in the previous section $\left({ }^{18} \mathrm{~F}\right.$-FDG PET/CT measurements).

We orthotopically implanted mice with A549 cells. We evaluated the success of orthotopic implantations as well as tumor volumes in all animals by periodic $\mathrm{CT}$ after implantation. When tumor volumes reached $>1 \mathrm{~mm}^{3}$, mice were treated with CDDP or erlotinib. These treatments were initiated on days 21 , 50, and 35 after implantation with A549, FT821, and PC-9 cells, respectively. Mice implanted with A549 cells were divided into four groups: control $[\mathrm{n}=4$, saline solution $(0.7 \mathrm{ml})$, single administration intraperitoneally]; CDDP [ $\mathrm{n}=4,7 \mathrm{mg} / \mathrm{kg}$ CDDP $(0.7 \mathrm{ml})$, single administration intraperitoneally]; control [ $\mathrm{n}=4$, $6 \%$ sulfobutylether- $\beta$-cyclodextrin (Captisol) (ChemScene, Monmouth Junction, NJ, USA) solution $(0.2 \mathrm{ml}) /$ day, oral administration 5 days/week]; and erlotinib $(n=4,25 \mathrm{mg} / \mathrm{kg}$ erlotinib dissolved in 6\% Captisol solution/day, oral administration 5 days/week) $(21,22)$. CDDP and erlotinib were obtained from Pfizer Japan, Inc. and Selleck Chemicals (Houston, TX, USA), respectively. Mice implanted with FT821 and PC-9 cells were similarly divided into four groups ( $\mathrm{n}=3 /$ group). After treatments, mice were evaluated by CT every third day.

Analysis of primary tumor volumes and $S U V_{\max }$. PET and CT images were analyzed using Inveon Research Workplace software (IRW; version 3.0; Siemens Healthcare). In all PET/CT datasets, the volume of interest (VOI) was defined manually around the primary tumor on $\mathrm{CT}$ images as the tumor volume. In fused PET images, the maximum standardized uptake value $\left(\mathrm{SUV}_{\max }\right)$ was calculated from the maximum voxel value $(\mathrm{Bq} / \mathrm{ml})$ in the VOI.

Histological examination of lymph nodes and lung metastasis. Among the mice examined by PET/CT and CT, all mice in each group were used for pathological analyses. We sacrificed mice and en bloc resected the bilateral lungs, trachea and bronchi, heart, esophagus, and mediastinal region of the mouse. Implanted tumors in the lungs were sectioned at a maximal cut and the lungs were cut into 5 to 61 - to 2 -mm-thick pieces in the minor axis direction and the mediastinum was cut in the longitudinal axis direction. These pieces were fixed by $10 \%$ formalin and embedded in paraffin. Paraffin sections stained with hematoxylin and eosin (H\&E) were examined by a Leica DM 2500 light microscopy (Leica Microsystems, Wetzlar, Germany) and the sizes of tumors were measured using the ruler of the microscope.

Statistical analysis. Tumor volumes and $\mathrm{SUV}_{\max }$ numbers in the control and CDDP groups were compared using the Mann-Whitney U test. Relationships between tumor volumes and $\mathrm{SUV}_{\max }$ were assessed using Spearman's test (SPSS software, version 20; IBM Inc., New York, NY, USA). A P-value of $<0.05$ was considered to indicate a statistically significant difference. 
A

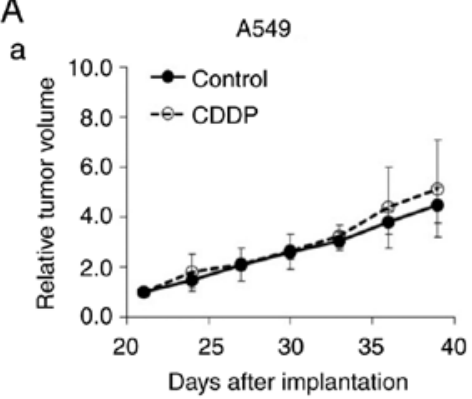

d

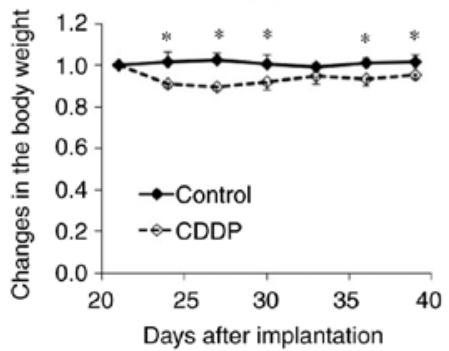

b
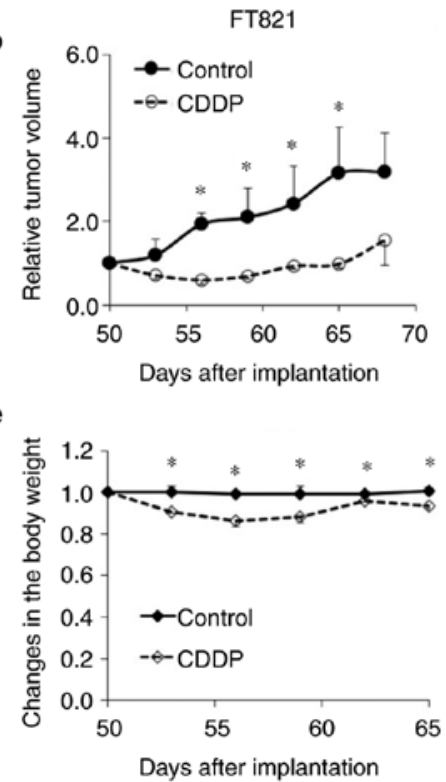

PC-9

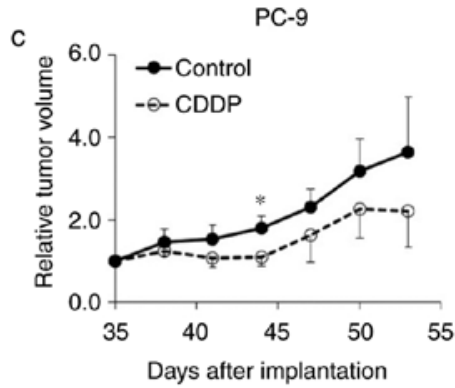

f

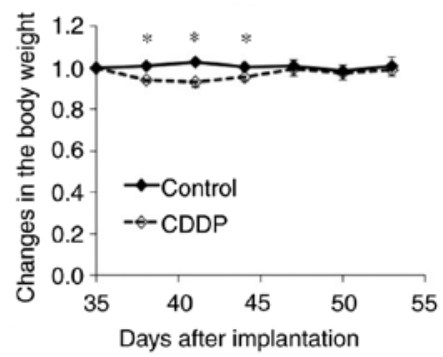

B
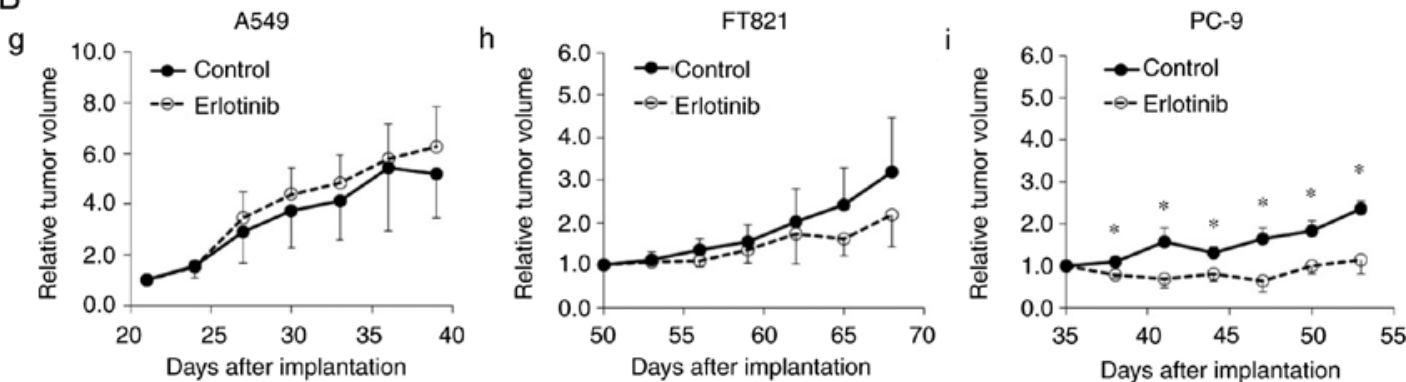

j
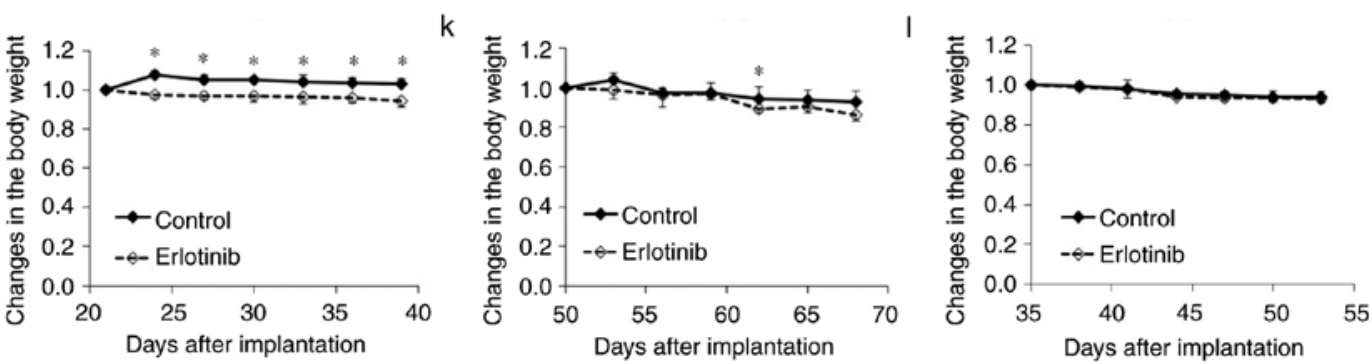

Figure 2. (A) Tumor volumes and body weights in mice implanted with: a and d, A549; b and e, FT821; or c and f, PC-9 cells and treated, as indicated, with CDDP or vehicle (control). (B) Tumor volumes and body weights in mice implanted with: $\mathrm{g}$ and $\mathrm{j}, \mathrm{A} 549$; $\mathrm{h}$ and $\mathrm{k}$, FT821; or i and 1, PC-9 cells and treated, as indicated, with erlotinib or vehicle (control). ${ }^{*} \mathrm{P}<0.05$, significant difference between control and CDDP groups.

\section{Results}

Monitoring responses using tumor volumes and $S U V_{\max }$. Coronary CT and PET/CT images of control and CDDP mice are presented in Fig. 1A. In both groups, CT scans detected a lung tumor in the left lung on day 20 after implantation. The sizes of tumors in both groups increased from day 20 to 50 . In coronal PET/CT images of control and CDDP mice, intense FDG uptake was observed in the tumors of both groups on day 30 and the intensity of FDG uptake increased from day 30 to 50. The tumor volumes and $\mathrm{SUV}_{\max }$ of the control and CDDP groups are displayed in Fig. 1B. Tumor volumes in both groups increased exponentially, and were similar in the CDDP and control groups from day 20 to 50 after implantation (Fig. 1B-a). SUV $V_{\max }$ increased from day 20 to 50 after implantation, with no significant difference between the control and CDDP groups (Fig. 1B-b). A correlation was observed between tumor volumes and $\mathrm{SUV}_{\max }$ in both groups (control: $r=0.76, \mathrm{P}<0.01$; CDDP: r=0.70, P<0.01; Fig. 1B-c).

Anticancer effects of CDDP and erlotinib in mice with A549-, FT821-, and PC-9-implanted tumors assessed by CT imaging. We examined the anticancer effects of CDDP in mice with A549-, FT821-, and PC-9-implanted tumors using CT imaging. The tumor volumes of A549 implants in the CDDP group were similar to those in the control group, confirming the results of the previous experiment (Fig. 2A-a). The tumor volumes of FT821 implants in the CDDP group were significantly smaller than those in the control group $(\mathrm{P}<0.05)$ (Fig. 2A-b). The tumor volumes of PC-9 implants in the CDDP group were 
A

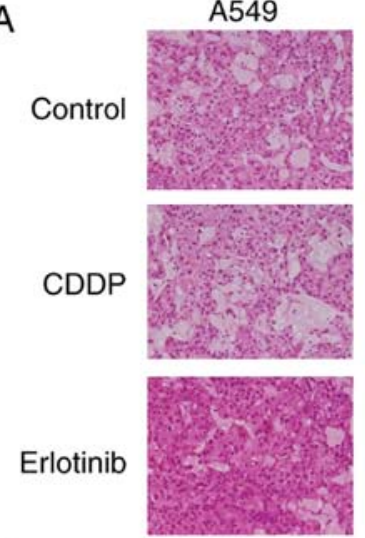

B

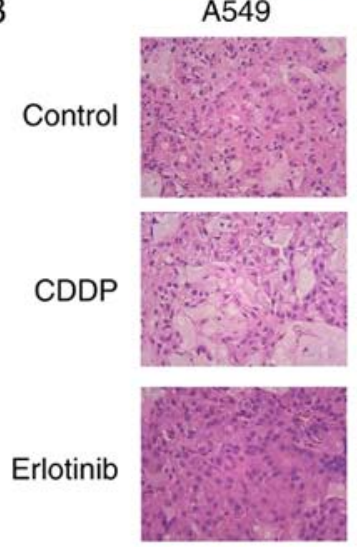

FT821
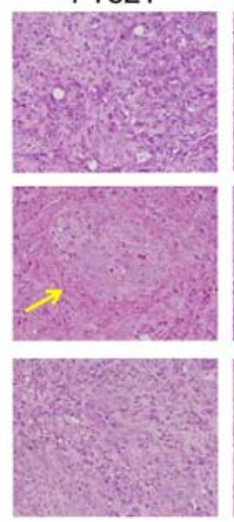

FT821
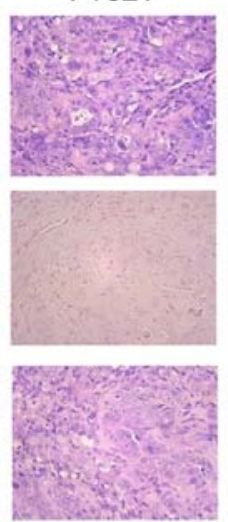

PC-9
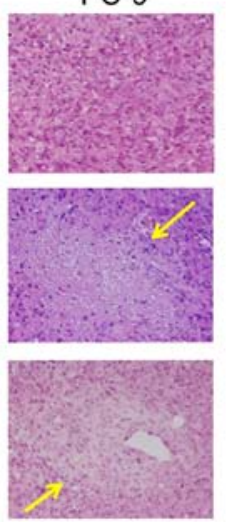

PC-9
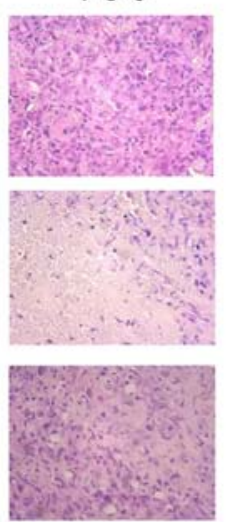

Figure 3. Histopathology of representative mice from control and treated (CDDP and erlotinib) groups. Hematoxylin and eosin (H\&E) staining magnified (A) $x 200$ and (B) $\times 400$. Yellow arrows show necrotic lesions.

smaller than those in the control group, and this difference was significant on day 44 only $(\mathrm{P}<0.05)$ (Fig. $2 \mathrm{~A}-\mathrm{c})$.

In the CDDP groups, the body weights of mice with A549 and FT821 implants were significantly lower than those of the respective control groups. In mice with PC-9 implants, this reduction in body weight was temporary (Fig. 2A, d-f).

We examined the anticancer effects of erlotinib in mice with A549-, FT821-, and PC-9-implanted tumors by CT imaging. The tumor volumes of A549 implants in the erlotinib group were similar to those in the control group (Fig. 2B-g). The tumor volumes of FT821 implants were slightly lower in the erlotinib group than in the control group (Fig. 2B-h). The tumor volumes of PC-9 implants were significantly lower in the erlotinib group than in the control group $(\mathrm{P}<0.05)$ (Fig. 2B-i).

The body weights of mice with A549 implants were significantly lower in the erlotinib group than in the control group (Fig. 2B-j). The body weights of mice with FT821 and PC-9 implants were similar with and without the erlotinib treatment (Fig. 2B, k and l).

Detection of lymph node metastases of the mediastinum and lung metastases using CT imaging and histopathology. Primary tumors were histopathologically confirmed in the lungs of all mice (Fig. 3). In the control groups, no necrotic lesions were observed in the tumors. In the CDDP groups, there were some necrotic lesions in mice with PC-9 and FT821 tumors, but not in those with A549 tumors. There were some
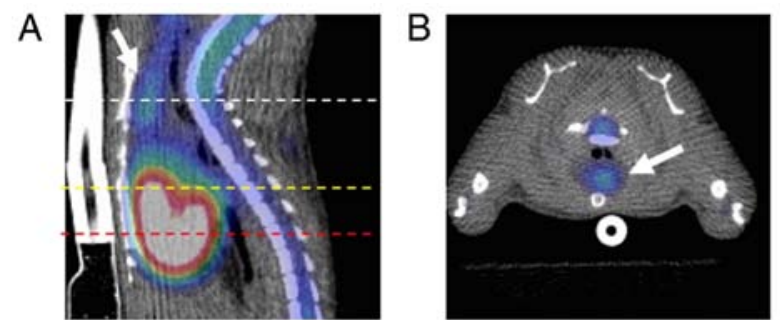

SUV

C

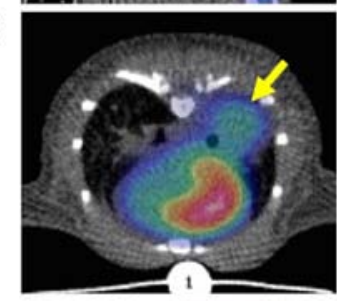

E
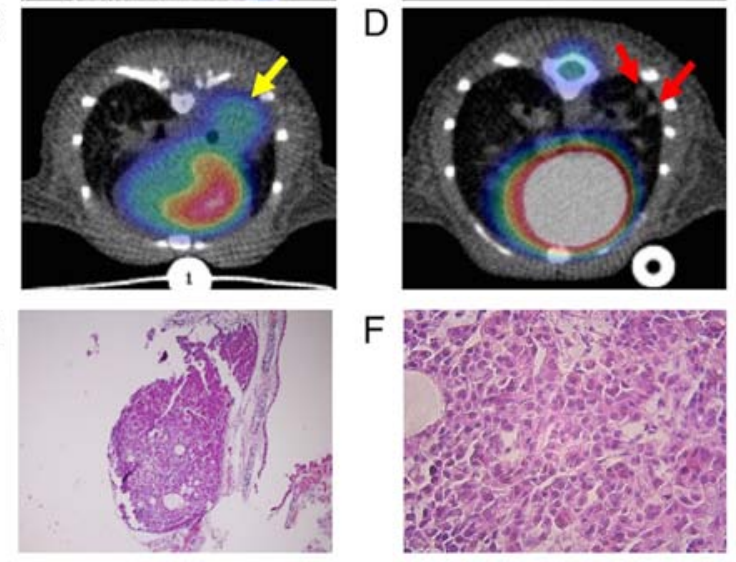

2.0

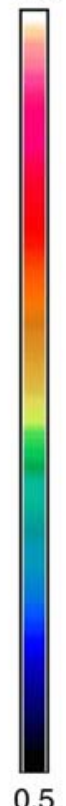

Figure 4. (A) A sagittal PET/CT image on day 50 after implantation of the mediastinum revealed A549 tumor FDG uptake; the white dotted line in the sagittal section indicates (B) the position of axial section FDG uptake by mediastinum metastases (white arrow), the yellow dotted line in the sagittal section indicates $(\mathrm{C})$ the position of axial section FDG uptake by the inoculate tumor (yellow arrow), the red dotted line in the sagittal section indicates (D) the position of axial section FDG uptake by lung metastases (red arrows). Histopathology confirmed lymph node metastasis of the mediastinum, with H\&E staining, magnified (E) x40 and (F) x400.

necrotic lesions in erlotinib-treated mice with PC-9, but not A549 or FT821 tumors.

Some mice exhibited the accumulation of FDG in the mediastinum in the control and CDDP groups. Representative sagittal and axial PET/CT images of the mediastinum on day 50 after implantation are revealed in Fig. 4A and B, with high FDG uptake in the mediastinum (white arrows). Histopathology revealed lymph node metastases of the mediastinum microscopically (Fig. 4E and F). In CT images, lung metastases were detectable, whereas ${ }^{18} \mathrm{~F}-\mathrm{FDG}$ uptake was not (Fig. 4D).

Metastases of the lung and mediastinal lymph nodes, as detected by $\mathrm{CT}$ and histopathology, in mice treated with or without CDDP and with erlotinib are presented in Table I. CT imaging detected lung metastases in A549-, but not FT821- or PC-9-implanted mice. CT imaging did not detect lymph node metastasis in any of the cell lines.

In the histopathological analysis, the frequencies of lung and lymph node metastases in A549-implanted mice, whose tumors were resistant to CDDP, were slightly higher with the CDDP treatment than in the controls (100 vs. $75 \%, 100$ vs. $75 \%$, for lung and lymph nodes, respectively). There were no lung metastases in the FT821-implanted mice, whose primary tumors had responded to CDDP, in the CDDP group, whereas they were detected in the control group (100\%). The lymph node metastasis rate of the mediastinum in the FT821-implanted mice was lower with the CDDP treatment than in the control group (66 vs. 100\%). The frequency of lung metastasis in the 


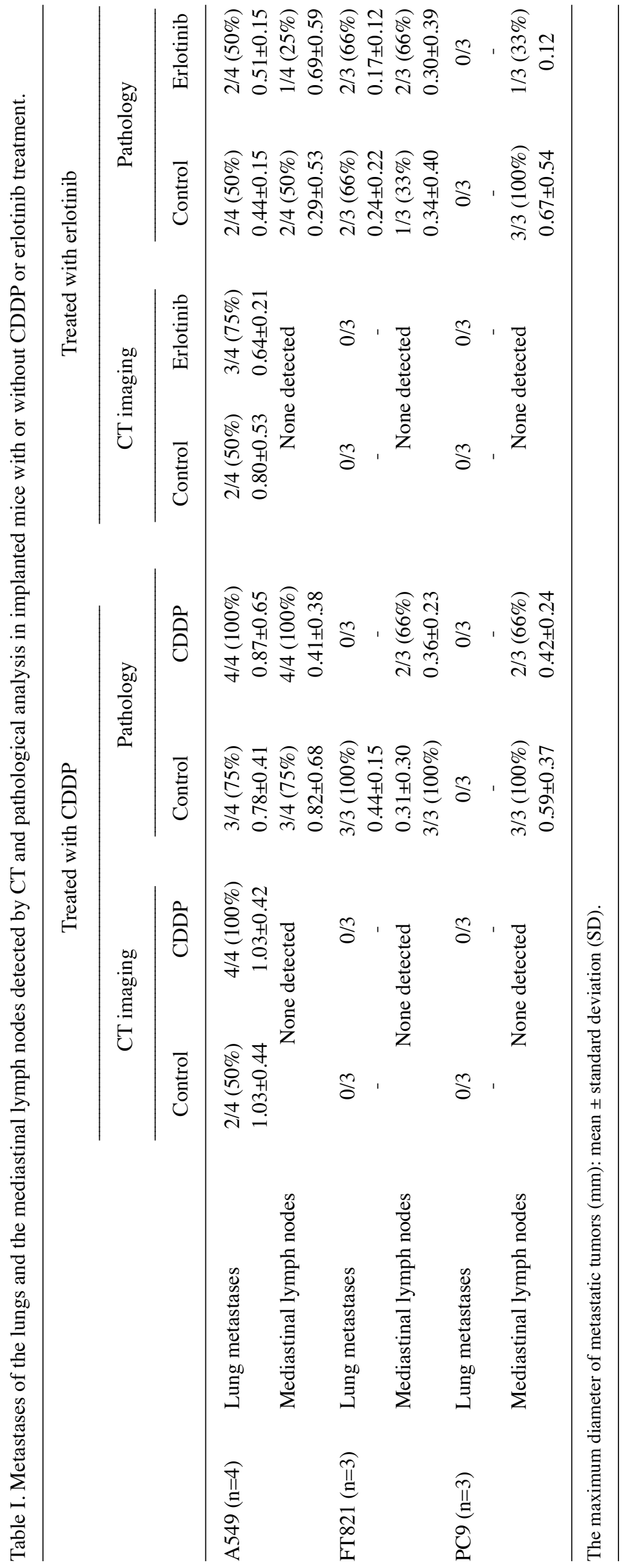


PC-9-implanted mice, in which CDDP had been slightly effective, was lower with the CDDP treatment than in the control group (66 vs. 100\%). In each cell line, the anticancer effects of CDDP for implanted tumors correlated with its effects on lung and lymph node metastases.

Regarding mice treated with or without erlotinib, the frequency of lymph node metastasis of the mediastinum in PC-9 implants, which were responsive to erlotinib, was lower with the erlotinib treatment than in the control group (33 vs. 100\%).

\section{Discussion}

Since many patients with NSCLC have lymphadenopathy and/or distant metastasis when diagnosed, more than $80 \%$ are potential beneficiaries of palliative systemic therapy (23). Palliative first-line chemotherapy was revealed to improve the quality of life and survival of advanced NSCLC patients due to newly introduced drugs and patient selection based on different histological subtypes and driver mutations. These selection parameters may identify the biology of malignancies and help predict drug efficacy (24). Recently developed molecular analyses for NSCLC have led to proposals for many new molecularly targeted drugs to treat $\operatorname{NSCLC}(3,4)$.

To provide suitable preclinical systems for evaluating the efficacy of these drugs, we established patient-like models of lung cancer metastasis by orthotopically implanting human NSCLC cell lines without thoracotomy (10-15). The models we described have the following advantages: i) The implantation procedure is simple and reproducible; ii) many tumor-implanted mice may be produced at once; iii) the procedure may be applied to many NSCLC cell lines; and iv) metastatic patterns, showing lymphatic metastases and/or distant metastases at high frequencies, are similar to those observed clinically in human lung cancer. However, the major disadvantage of these models is that the tumors, including primary and metastatic, were difficult to monitor continuously and reproducibly, and may only be assessed at necropsy. We previously evaluated the utility of ${ }^{18} \mathrm{~F}$-FDG PET-CT to non-invasively and repeatedly monitor the anticancer effects of CDDP in an orthotopic lung cancer model using Ma44-3 cells. We demonstrated the significant inhibition of tumor growth by the CDDP treatment, and also revealed that tumor volumes and $\mathrm{SUV}_{\max }$ correlated in these mice $\left(r^{2}=0.67, P=0.048\right)(19)$. In the present study, we evaluated the utility of ${ }^{18} \mathrm{~F}$-FDG PET-CT to monitor the anticancer effects of CDDP in an orthotopic model using another lung cancer cell line, A549. Tumor volumes and $\mathrm{SUV}_{\max }$ were similar in the CDDP and control groups, and tumor volume correlated with $\mathrm{SUV}_{\max }$ in this A549 model (control: $\mathrm{r}=0.76$, $\mathrm{P}<0.01$; CDDP: $\mathrm{r}=0.70, \mathrm{P}<0.01)$. Thus, it was demonstrated that ${ }^{18}$ F-FDG PET/CT discriminates between CDDP-responsive and -unresponsive tumors, Ma44-3 and A549, respectively. Since $\mathrm{SUV}_{\max }$ assessed by PET correlated with tumor volumes using CT imaging, we subsequently used CT only to estimate the anticancer effects of CDDP in FT821- and PC-9-implanted models and of erlotinib in models using all cell lines.

Our histological results correlated with anticancer effects. FT821 and PC-9, but not A549 tumors exhibited necrotic lesions with CDDP. Therefore, CT imaging detected the anticancer effects of CDDP, a major cytotoxic anticancer drug that is intravenously administered. We then used erlotinib, a molecularly targeted drug that is orally administered. PC-9 tumors were very responsive to erlotinib, while A549 and FT821 tumors were not. Histological results correlated with these anticancer effects; PC-9 exhibited necrotic lesions, whereas A549 and FT821 did not. PC-9 cells have an EGFR mutation. The CT monitoring system may detect the anticancer effects of molecularly targeted drugs or orally administered drugs such as erlotinib. Moreover, anticancer effects correlated with the frequencies of lung and lymph node metastases. Our orthotopic models of lung cancer revealed frequent metastases to the lymph nodes, similar to the metastatic patterns of lung cancer observed in clinical settings. We found that PET/CT imaging detected lymph node metastases of the mediastinum, while CT imaging detected lung metastases. In the present study, all cell lines, A549, FT821, and PC-9, exhibited lymph node metastasis of the mediastinum, as identified histopathologically. CT imaging did not detect lymph node metastasis of the mediastinum due to poor contrast between the normal mediastinum tissue and lymph node metastatic tumors. However, ${ }^{18}$ F-FDG PET/CT imaging detected FDG uptake in lymph node metastases of the mediastinum. Therefore, the latter method may be used to non-invasively and repeatedly monitor the anticancer effects of agents in lymph node metastases of the mediastinum. In patients, ${ }^{18} \mathrm{~F}-\mathrm{FDG}$ PET/CT is useful for the diagnosis of mediastinal lymph node metastasis and estimating the responses of lymph node metastases to anticancer drugs (16). The present results demonstrated that CT imaging may be used to non-invasively and repeatedly monitor the anticancer effects of less specific drugs, such as CDDP, and molecularly targeted drugs, including erlotinib, on several different lung cancers.

In conclusion, we herein demonstrated that PET/CT and CT imaging of our orthotopic SCID mouse models of lung cancer enabled the non-invasive and repeated monitoring of the anticancer efficacies of CDDP and erlotinib. These effects were detected not only in implanted tumors, but also in mediastinal lymph node and lung metastases. These methods may be applied to a number of NSCLC cell lines and anticancer drugs. Therefore, our models have potential as fundamental tools for the design and development of new therapies for cancer.

\section{Acknowledgements}

We would like to thank Professor Seiji Yano (Professor and Chairman, Division of Medical Oncology, Cancer Research Institute, Kanazawa University) for providing the PC-9 cell line.

\section{Funding}

The present study was supported by a Grant-in-Aid for Scientific Research from the Ministry of Education, Culture, Sports, Science and Technology (24659634), Japan.

\section{Availability of data and materials}

The datasets used during the present study are available from the corresponding author upon reasonable request. 


\section{Authors' contributions}

KKo conceived and designed the study. TO, HT and HF acquired the data. TO, HT, KKa, HO and HM analyzed the data. TO and KKo drafted the manuscript and were involved in the conception of the study. All authors read and approved the manuscript and agree to be accountable for all aspects of the research in ensuring that the accuracy or integrity of any part of the work are appropriately investigated and resolved.

\section{Ethics approval and consent to participate}

All experimental protocols were reviewed and approved by the Animal Research Committee of The University of Tokushima, Japan.

\section{Patient consent for publication}

Not applicable.

\section{Competing interests}

The authors declare that they have no competing interests.

\section{References}

1. Stinchcombe TE, Bogart J, Veeramachaneni NK, Kratzke R and Govindan R: Annual review of advances in non-small cell lung cancer research: A report for the year 2010. J Thorac Oncol 6: 1443-1450, 2011

2. Horner MJ, Ries LAG, Krapcho M, Neyman N, Aminou R, Howlader N, Altekruse SF, Feuer EJ, Huang L, Mariotto A, et al (eds): National Cancer Institute, SEER cancer statistics review, 1975-2006. http://seer.cancer.gov/csr/1975_2006. Accessed November, 2009.

3. Ding L, Getz G, Wheeler DA, Mardis ER, McLellan MD Cibulskis K, Sougnez C, Greulich H, Muzny DM, Morgan MB et al: Somatic mutations affect key pathways in lung adenocarcinoma. Nature 455: 1069-1075, 2008.

4. Cancer Genome Atlas Research Network: Comprehensive genomic characterization of squamous cell lung cancers Nature 489: 519-525, 2012.

5. Francia G, Cruz-Munoz W, Man S, Xu P and Kerbel RS: Mouse models of advanced spontaneous metastasis for experimental therapeutics. Nat Rev Cancer 11: 135-141, 2011.

6. Paget S: The distribution of secondary growths in cancer of the breast. Lancet 133: 571-573, 1889.

7. Wilmanns C, Fan D, O'Brian CA, Bucana CD and Fidler IJ: Orthotopic and ectopic organ environments differentially influence the sensitivity of murine colon carcinoma cells to doxorubicin and 5-fluorouracil. Int J Cancer 52: 98-104, 1992.

8. Fidler IJ: Models for spontaneous metastasis. Cancer Res 66 9787, 2006

9. Peterson JK and Houghton PJ: Integrating pharmacology and in vivo cancer models in preclinical and clinical drug development. Eur J Cancer 40: 837-844, 2004.
10. Miyoshi T, Kondo K, Ishikura H, Kinoshita H, Matsumori Y and Monden Y: SCID mouse lymphogenous metastatic model of human lung cancer constructed using orthotopic inoculation of cancer cells. Anticancer Res 20: 161-163, 2000

11. Ishikura H, Kondo K, Miyoshi T, Kinoshita H, Hirose T and Monden Y: Artificial lymphogenous metastatic model using orthotopic implantation of human lung cancer. Ann Thorac Surg 69: 1691-1695, 2000.

12. Ishikura H, Kondo K, Miyoshi T, Kinoshita H, Takahashi Y, Fujino $\mathrm{H}$ and Monden Y: Suppression of mediastinal metastasis by uracil-tegafur or cis-diamminedichloroplatinum(II) using a lymphogenous metastatic model in a human lung cancer cell line. Clin Cancer Res 7: 4202-4208, 2001.

13. Fujino H, Kondo K, Miyoshi T, Ishikura H, Takahashi Y, Sawada N, Hirose Y, Takizawa H, Nagao T, Sakiyama S, et al: Establishment of patient-like SCID mouse model by orthotopically implanting primary cultured cells from surgically-resected lung cancer tissues. Oncol Rep 10: 1709-1715, 2003.

14. Fujino H, Kondo K, Ishikura H, Maki H, Kinoshita H, Miyoshi T, Takahashi Y, Sawada N, Takizawa H, Nagao T, et al: Matrix metalloproteinase inhibitor MMI-166 inhibits lymphogenous metastasis in an orthotopically implanted model of lung cancer. Mol Cancer Ther 4: 1409-1416, 2005.

15. Takizawa H, Kondo K, Toba H, Kenzaki K, Sakiyama S and Tangoku A: Fluorescence diagnosis of lymph node metastasis of lung cancer in a mouse model. Oncol Rep 22: 17-21, 2009.

16. Sauter AW, Spira D, Schulze M, Pfannenberg C, Hetzel J, Reimold M, Klotz E, Claussen CD and Horger MS: Correlation between $\left[{ }^{18} \mathrm{~F}\right]$ FDG PET/CT and volume perfusion CT in primary tumours and mediastinal lymph nodes of non-small-cell lung cancer. Eur J Nucl Med Mol Imaging 40: 677-684, 2013.

17. Walker CM, Chung JH, Abbott GF, Little BP, El-Sherief AH, Shepard JA and Lanuti M: Mediastinal lymph node staging: From noninvasive to surgical. AJR Am J Roentgenol 199: W54-W64, 2012.

18. Silvestri GA, Gould MK, Margolis ML, Tanoue LT, McCrory D, Toloza E and Detterbeck F; American College of Chest Physicians: Noninvasive staging of non-small cell lung cancer: ACCP evidenced-based clinical practice guidelines (2nd edition). Chest 132 (3 Suppl): 178S-201S, 2007.

19. Mokhtar M, Kondo K, Takizawa H, Ohtani T, Otsuka H, Kubo H, Kajiura K, Nakagawa Y, Kawakami Y, Yoshida M, et al: Non-invasive monitoring of anticancer effects of cisplatin on lung cancer in an orthotopic SCID mouse model using $\left[{ }^{18} \mathrm{~F}\right] \mathrm{FDG}$ PET-CT. Oncol Rep 31: 2007-2014, 2014.

20. Wang W, Li Q, Yamada T, Matsumoto K, Matsumoto I, Oda M, Watanabe G, Kayano Y, Nishioka Y, Sone S and Yano S: Crosstalk to stromal fibroblasts induces resistance of lung cancer to epidermal growth factor receptor tyrosine kinase inhibitors. Clin Cancer Res 15: 6630-6638, 2009.

21. Ishikawa D, Takeuchi S, Nakagawa T, Sano T, Nakade J, Nanjo S, Yamada T, Ebi H, Zhao L, Yasumoto K, et al: mTOR inhibitors control the growth of EGFR mutant lung cancer even after acquiring resistance by HGF. PLoS One 8: e62104, 2013.

22. Nanjo S, Ebi H, Arai S, Takeuchi S, Yamada T, Mochizuki S, Okada Y, Nakada M, Murakami T and Yano S: High efficacy of third generation EGFR inhibitor AZD9291 in a leptomeningeal carcinomatosis model with EGFR-mutant lung cancer cells. Oncotarget 7: 3847-3856, 2016.

23. Johnson DH, Schiller JH and Bunn PA Jr: Recent clinical advances in lung cancer management. J Clin Oncol 32: 973-982, 2014.

24. Noonan KL, Ho C, Laskin J and Murray N: The influence of the evolution of first-line chemotherapy on steadily improving survival in advanced non-small-cell lung cancer clinical trials. J Thorac Oncol 10: 1523-1531, 2015. 